\title{
Lettre posthume à Gesualdo Bufalino
}

Lettera postuma a Gesualdo Bufalino

Posthumous Letter to Gesualdo Bufalino

Jacques Michaut-Paternò

\section{OpenEdition \\ Journals}

Édition électronique

URL : http://journals.openedition.org/cei/6447

DOI : 10.4000/cei.6447

ISSN : 2260-779X

Éditeur

UGA Éditions/Université Grenoble Alpes

Édition imprimée

ISBN : 978-2-37747-173-7

ISSN : 1770-9571

Référence électronique

Jacques Michaut-Paternò, « Lettre posthume à Gesualdo Bufalino », Cahiers d'études italiennes [En ligne], 30 | 2020, mis en ligne le 01 mars 2020, consulté le 15 septembre 2020. URL : http:// journals.openedition.org/cei/6447

Ce document a été généré automatiquement le 15 septembre 2020

(c) ELLUG 


\title{
Lettre posthume à Gesualdo Bufalino
}

\author{
Lettera postuma a Gesualdo Bufalino \\ Posthumous Letter to Gesualdo Bufalino
}

Jacques Michaut-Paternò

Paris, 11 novembre 2019

Cher Gesualdo Bufalino,

Vous ai-je servi ou vous ai-je trahi ? Ai-je été l'ingénieur, le cambrioleur de coffre-fort, l'amoureux, le mystique, ce « quelque chose de semblable à celui qui aide un aveugle à traverser la rue », ce passeur dont vous parlez dans vos nombreuses interrogations sur la traduction, voire le double français d'une dédicace flatteuse ? L'amoureux sans aucun doute, celui qui, chaque été, quittant le refuge d'une petite île éolienne, gagnait, une fois franchi le bras de mer qui sépare Lipari de Milazzo, ce fabuleux triangle de lumière et de deuil dont vous alliez lui révéler peu à peu les secrets et arrivait jusqu'à un angle doré tapissé de caroubiers et de figuiers de barbarie au pied des Iblei, à Comiso, votre tanière. Je sonnais fébrilement au 26 de la via Mancini, vous m'accueilliez un grand sourire aux lèvres, le regard à la fois scrutateur et bienveillant, un brin malicieux, derrière les épaisses lunettes dont le cadre austère dans les premiers temps m'impressionnait mais qui concentrait dans son apparente sévérité tant d'indulgence et de bonté que je finis par m'attacher à ce qui n'était plus une barrière comme j'aurais $\mathrm{pu}$ le croire mais une invitation, une ouverture sur un autre monde, votre monde. Installés dans le demi-jour rafraîchissant d'un salon bibliothèque où figurait en bonne place le célèbre portait de Baudelaire photographié par Nadar, la conversation s'engageait, au fil des ans plus familière, vous toujours d'une délicate prévenance, moi pris sous le charme, dans les premiers temps empêtré dans les filets d'une ridicule timidité puis mis en confiance par votre attention, votre simplicité, votre gentillesse naturelle. Nous abordions les thèmes les plus divers puis en venions au texte de l'œuvre en cours de traduction, la plupart du temps des mises au point de remarques dont vous m'aviez précédemment expédié la liste au fur et à mesure où je vous postais les avancées de mon travail, des précisions et des choix sur lesquels nous tombions 
immédiatement d'accord. Votre connaissance intérieure de la langue française, que votre longue fréquentation de Baudelaire et de Toulet entre autres, vos innombrables lectures, votre profonde imprégnation de culture française rendaient incomparable, facilitait grandement notre collaboration, la recherche de la traduction la plus juste, de l'impact du mot, de l'harmonie du phrasé. Autant de conditions on ne peut plus idéales pour un traducteur qui me donnaient l'illusion de recréer ce que j'aurais tant aimé créer. Dans mes hésitations ou mes doutes elles étaient la garantie d'une précieuse osmose entre nos deux cheminements, le vôtre celui de créateur, le mien celui d'humble recréateur, plus exactement d'interprète, à l'image du musicien ou du chanteur qui s'étant imprégné de la partition du compositeur tente d'en restituer sinon l'âme, ce qui tient du miracle, du moins l'image, impalpable reflet d'une autre réalité. Je sentais mieux encore que je ne comprenais ce que vous vouliez dire car votre langue infiniment ondoyante entre éclat et secret me fascinait, parlait à ma sensibilité, disait ce que je voulais dire, exprimait ce que je voulais écrire. La mienne, muselée dans le carcan d'une grammaire rigide, enserrée dans un filet de sonorités prisonnières d'un accent tonique irrémissiblement final, cherchait, difficilement parfois, à se lover dans votre musicalité. Y parvint-elle? Parvient-on d'ailleurs jamais à une parfaite adéquation? Tentative plus ingrate encore en poésie à laquelle Nabokov ne croyait guère puisqu'il conseillait prudemment de s'en tenir à un neutre mot à mot. Votre expérience baudelairienne fut en ce sens un redoutable et admirable pari.

Le dicton traduttore-traditore est un raccourci cruel et mensonger. Vous avez écrit que la traduction était plus qu'un exercice: un geste d'ascèse et d'amour. Il y entre effectivement beaucoup de sacrifice de soi et de don à l'autre, un sacrifice qui n'est pas privation ou amputation mais exigence et accomplissement, un don à l'autre qui n'est pas dépossession ou auto-négation mais offrande et complémentarité. Le hasard qui me conduisit un jour à vous n'était pas innocent, mais l'est-il jamais? Il est l'aboutissement d'une nécessité, sa concrétisation dans l'ordre des êtres et des choses, le fruit d'une recherche mûrissant entre conscience et inconscience. Lorsque j'eus pour la première fois entre les mains, moi qui alors n'avais encore rien lu de vous, L'Uomo invaso, je découvris dans vos récits, dans la matière qui en constituait la trame, dans la façon dont celle-ci se nouait, quelque chose dont j'étais à mon tour envahi, une façon d'être dans le monde et de le regarder, une manière d'en guetter la respiration, d'en épouser les mouvements. Ce que je lisais prolongeait ma réflexion, concrétisait en quelque sorte mes pensées. Je sortais d'un univers russe, celui de mes traductions antérieures, extrême, convulsif, irrationnel, démesuré, et me retrouvais sur le seuil d'un autre univers d'apparente clarté, d'évidente harmonie, de relative mesure, méditerranéen comme la mer qui le baigne mais, comme elle, traversé de courants redoutables, à la fois éblouissant de lumière et inquiétant de ténèbres. L'un et l'autre dans le fond n'étaient pas aussi opposés que cela malgré leur apparent éloignement, leurs contrastes, leurs incompatibilités premières: le décor changeait, le ciel, la nature, l'histoire, le destin, mais l'énigme restait la même, son message tout aussi indéchiffrable, son angoisse tout aussi poignante. Je n'étais donc pas désorienté, simplement placé dans une autre chambre de la grande maison qu'est l'âme humaine. Ce n'était pas à proprement parler une nouvelle expérience, plutôt la suite d'une quête. On ne traduit dignement que ce qu'on voudrait exprimer soi-même mais que les exigences de la perfection et les contingences du talent conseillent de tenir pudiquement secret. Et c'est ainsi que pas à pas, de livre en livre pénétrant au cœur de votre monde j'ai appris, guidé par vos soins, à «cambrioler votre coffre-fort». Les 
chercheurs et les commentateurs qui un jour voudront se pencher sur la traduction française de vos œuvres pour examiner et analyser cet étrange phénomène qu'est le passage d'une langue à l'autre auront pour ce qui me concerne une aide précieuse : vos indications consignées dans les feuillets que vous m'adressiez, listes pointilleuses et détaillées de vos remarques et de vos suggestions. Elles éclaireront en partie un processus de translation à la limite irréductible à toutes les théories échafaudées avec le concours des sciences nouvelles et le recours à la psychanalyse. Elles en codifieront les mécanismes mais n'en épuiseront pas, loin de là, la complexité et au bout du compte se heurteront à un mystère que le formalisme desséchant d'une analyse «sans divinité, sans inspiration " pour reprendre le cri lancé par le poète russe Alexandre Blok peu de temps avant de mourir en 1921 - et le chaos révolutionnaire de son époque n'est pas sans préfigurer l'effondrement d'un vieux monde devenu «musée d'ombres» - ne pourra jamais percer. Car c'est bien en dernier ressort de «divinité» et d'« inspiration » qu'il s'agit quand on parle de traduction et vous l'avez senti mieux que quiconque, vous qui étiez des deux côtés d'une barrière difficilement franchissable : la langue, sa musique, son " âme ». Vous avez enfin, là où vous êtes, la réponse à toutes les questions accumulées au fil de vos œuvres. Là où nous sommes nous les cherchons inlassablement. Ceux de vos livres que les «ingénieurs amoureux » de demain auront encore à faire passer de la langue de Dante à celle de Molière sans le concours miraculeux de votre regard et de vos soins, exigeront de leur part une piété et une humilité plus grandes encore. Mais ce n'est qu'à ce prix que le lecteur français pourra, dans l'obscurité du monde à venir, discerner l'étoile prophétique de votre œuvre et les rayons de votre espérance.

\section{AUTEUR}

JACQUES MICHAUT-PATERNÒ

Traducteur 